\title{
Intraocular pressure associations with refractive error and axial length in children
}

\author{
A J Lee, S-M Saw, G Gazzard, A Cheng, D T H Tan
}

Br J Ophthalmol 2004;88:5-7

\begin{abstract}
Aim: To assess whether intraocular pressure (IOP) is associated with refractive error or axial length in children. Methods: Of subjects from the Singapore Cohort Study of the Risk Factors for Myopia (SCORM), 636 Chinese children aged 9-11 years from two elementary schools underwent non-contact tonometry, cycloplegic autorefraction, and Ascan biometry during 2001. For analyses, refractive error was categorised into four groups; hypermetropia (spherical equivalent refraction (SE) $\geqslant+1.0 D)$, emmetropia $(-0.5 \mathrm{D}<\mathrm{SE}<+1 . \mathrm{OD})$, low myopia $(-3.0 \mathrm{D}<\mathrm{SE} \leqslant-0.5 \mathrm{D})$ and high myopia (SE $\leqslant-3.0 D)$.

Results: Of the 636 children examined, $50.6 \%$ were male. The mean IOP was 16.6 (SD 2.7) $\mathrm{mm} \mathrm{Hg}$. There were no significant IOP differences between low (mean IOP $=16.4$ $(2.8) \mathrm{mm} \mathrm{Hg}$ ) or high myopes $(16.7(2.5) \mathrm{mm} \mathrm{Hg})$ and emmetropes (16.7 (2.9) $\mathrm{mm} \mathrm{Hg}$ ), $p=0.57$. IOP was not correlated with spherical equivalent refraction (Spearman correlation, $r=0.009)$ or axial length $(r=0.030)$. In regression analyses adjusting for diastolic blood pressure, neither spherical equivalent (regression coefficient $=0.014$ ) nor axial length (regression coefficient $=0.027$ ) were significantly associated with IOP.

Conclusion: These findings do not support an association between IOP and refractive error or axial length in children. This questions postulated roles of IOP in the pathogenesis of myopia.
\end{abstract}

\footnotetext{
I
} ntraocular pressure (IOP) is one of several factors ${ }^{1}$ implicated in the pathogenesis of myopia. Elevated IOP is hypothesised to impose scleral stress and creep, resulting in axial elongation with scleral stretch. ${ }^{2}$ Several clinic based studies have evaluated the relation between IOP and myopia in children, based on the premise that school myopia occurs predominantly because of an increase in axial length (AL). Although some studies ${ }^{3-5}$ have documented a positive association, others ${ }^{67}$ have questioned the temporal relation between IOP and myopia in children. Also, clinical trials of ocular hypotensives have had unconvincing effects on halting the progression of myopia. ${ }^{89}$ Most population based studies in adults have however found significant relations between IOP and refractive error ${ }^{1011}$ or myopia, ${ }^{12}{ }^{13}$ although disputed by a few clinic based studies. ${ }^{14} 15$ The cross sectional nature of these studies in adults precludes ascertainment of the causeeffect relation between myopia and IOP.

In light of these factors that question whether IOP causes myopia, we aimed to assess whether IOP is associated with refractive error or AL using data collected from a large sample of Singaporean Chinese schoolchildren.

\section{PATIENTS AND METHODS}

The Singapore Cohort Study of the Risk Factors for Myopia (SCORM) recruited children aged 7-9 years during 1999. The methods of this study have been described elsewhere. ${ }^{16}$
Children with serious systemic diseases, ocular conditions including congenital cataract or a syndrome associated with myopia, or those with allergies to or who refused instillation of eye drops were excluded. The ethics committee, Singapore Eye Research Institute, and the Ministry of Education approved this study. Examination protocols followed the tenets of the Declaration of Helsinki and written informed consent was obtained from the parents.

Chinese children from two schools were recruited into the study. Of 698 Chinese children in this study, 636 (91.1\%) had refractive error and IOP measurements. Rates of myopia in participating children $(32.0 \%)$ were similar to those who did not $(32.0 \%), p=0.55$. Chinese children were selected to determine IOP associations in an ethnically homogeneous population.

Non-contact tonometry (NT-3000; Nidek Co, Tokyo, Japan) was performed on both eyes, with the right eye measured first, by a medically trained observer. Measurements adhered to protocols; the child was seated, with eyes in the primary position, eyelashes or eyelids did not obscure the applanation circle, and the alignment spot was centred and focused in the alignment circle before applanation. One reading (digitised) was taken.

After instillation of $0.5 \%$ oxybuprocaine (proparacaine), three drops of $1 \%$ cyclopentolate were administered 5 minutes apart to induce cycloplegia in each eye. After 45 minutes, five consecutive refraction and corneal curvature measurements were obtained by one of two autokeratorefractometers (RK5; Canon Inc, Tochigiken, Japan). Measurements of AL, anterior chamber depth (ACD), crystalline lens thickness (LT), and vitreous chamber depth (VCD) were performed using one of two ultrasound biometry machines (Echoscan US-800; Nidek Co, Tokyo, Japan; probe frequency $10 \mathrm{mHz}$ ). Six AL measurements were averaged; the SD of these six readings was $<0.12 \mathrm{~mm}$. The child's parents completed the questionnaire 2 weeks before the eye examinations. Information collected included total family income, parental level of education, and history of myopia and glaucoma. Seated blood pressure (BP) (Omron HEM 705 LP) was also measured.

\section{Data analysis}

The IOP of right and left eyes were highly correlated (Pearson's correlation coefficient $=0.75$ ). Refraction was analysed as spherical equivalent (SE), calculated as sphere plus negative half cylinder. Refractive error was stratified into four categories: hypermetropia $(\mathrm{SE} \geqslant 1.0 \mathrm{D})$, emmetropia $(-0.5 \mathrm{D}<\mathrm{SE}<+1.0 \mathrm{D})$, low myopia $(-3.0 \mathrm{D}<\mathrm{SE} \leqslant-0.5 \mathrm{D})$, and high myopia ( $\mathrm{SE} \leqslant-3.0 \mathrm{D})$. Astigmatism was defined by cylinder at least $-0.5 \mathrm{D}$.

Correlations between IOP, refraction, and the biometry parameters of the right and left eyes separately were calculated using the Spearman correlation coefficient. Results were the same when the left eye was analysed, thus right eye data are presented. Two multiple linear regression models were constructed with IOP as the dependent variable and SE or AL as the main covariates, controlling for BP. Data analyses were conducted using STATA (version 7.0). ${ }^{17}$ 


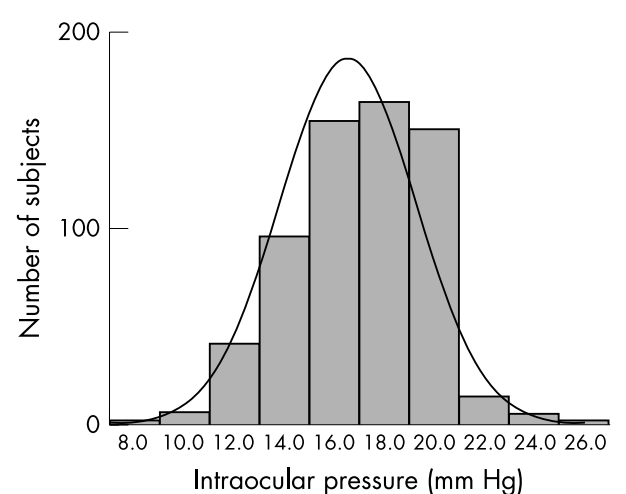

Figure 1 Distribution of right eye intraocular pressure in Chinese schoolchildren $(\mathrm{n}=636)$.

\section{RESULTS}

Of 636 children examined; $50.6 \%$ were male; $54.6 \% 9$ years, $33.3 \% 10$ years, and $12.1 \% 11$ years. The mean age (SD) of females was $9.6(0.74)$ years and of males was $9.6(0.78)$ years. The mean SE of females was - 1.58D (SD 2.09) and of males $-1.70 \mathrm{D}$ (2.27). Figure 1 illustrates the distribution of right eye IOP in this population. The mean IOP was 16.6 (2.7) $\mathrm{mm} \mathrm{Hg}$, and the median was $17.0 \mathrm{~mm} \mathrm{Hg}$.

Table 1 presents right eye IOP by age, sex, and right eye refractive error status. No significant differences in IOP were found between age groups $(p=0.71)$ and sex $(p=0.32)$. No relations were found between IOP and parental income, parental level of education or parental history of myopia, glaucoma or hypertension.

The mean IOP in children with hyperopia (16.7 mm Hg), emmetropia (16.7 $\mathrm{mm} \mathrm{Hg}$ ), low myopia (16.4 $\mathrm{mm} \mathrm{Hg}$ ), and high myopia ( $16.7 \mathrm{~mm} \mathrm{Hg}$ ) were similar. The scatter plot for the relation between IOP and refractive error of right eyes (fig 2), emphasises that no correlation exists (Spearman correlation $r=0.009)$. The mean IOP of children with astigmatism ( $16.5 \mathrm{~mm} \mathrm{Hg}$ ) was similar to children without ( $16.6 \mathrm{~mm} \mathrm{Hg}$ ), $\mathrm{p}=0.62$.

Of ocular parameters, IOP was neither correlated with AL (Spearman correlation $r=0.030$ ) (fig 3), nor with VCD $(r=0.018)$, LT $(r=-0.009)$, ACD $(r=0.054)$, or corneal radius of curvature $(r=0.029)$. Correlations were found between IOP and systolic $(r=0.13)$ or diastolic $(r=0.13)$ blood pressure. In regression analyses adjusting for diastolic $\mathrm{BP}$, neither SE (regression coefficient $=0.014$ ) nor $\mathrm{AL}$ (regression coefficient $=0.027)$ was significantly associated with IOP.

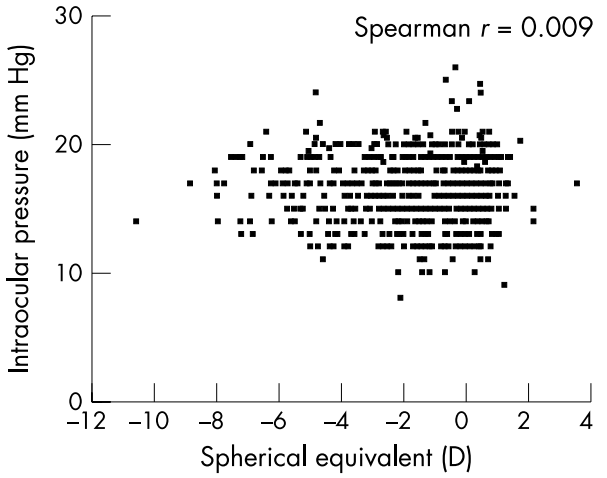

Figure 2 Correlation between intraocular pressure and spherical equivalent (right eye data).

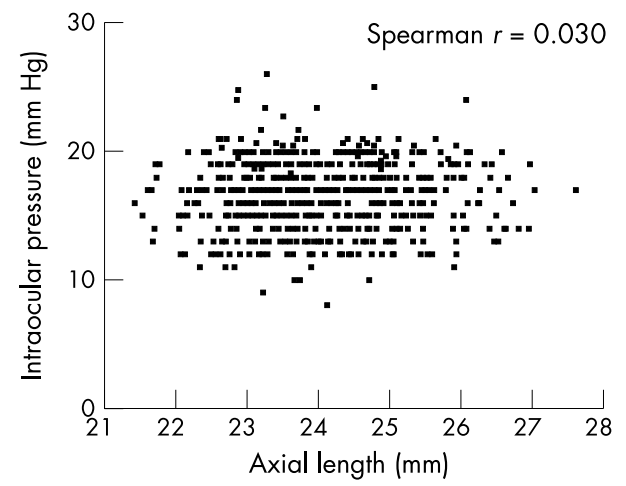

Figure 3 Correlation between intraocular pressure and axial length (right eye data).

\section{DISCUSSION}

Our findings question the role of elevated IOP in the development of axial elongation and myopia in young children. However, we cannot comment upon the role or temporal relation of elevated IOP in adult myopes. The lack of association between IOP and AL, an ocular parameter strongly associated with myopia, provides some support for those theories of myopia pathogenesis acting independently of IOP. Retinal defocus may drive abnormal eye growth in children.

We evaluated a large random sample of Chinese children at an age of rapid growth, and included ocular biometry. The children tolerated non-contact tonometry, which was quick, accurate $^{18}$ and relatively observer independent. Inherent

\begin{tabular}{|c|c|c|c|c|}
\hline & \multirow[b]{2}{*}{ No } & \multicolumn{2}{|c|}{ Right eye IOP (mm Hg) } & \multirow[b]{2}{*}{ p Value } \\
\hline & & Mean (SD) & Median (range) & \\
\hline Total & 636 & $16.6(2.7)$ & $17.0(8.0-26.0)$ & \\
\hline \multicolumn{5}{|l|}{ Age (years) } \\
\hline 9 & 347 & $16.6(2.7)$ & $17.0(8.0-26.0)$ & 0.71 \\
\hline 10 & 212 & $16.5(2.7)$ & $17.0(10.0-25.0)$ & \\
\hline 11 & 77 & $16.4(2.8)$ & $17.0(10.0-24.7)$ & \\
\hline \multicolumn{5}{|l|}{ Sex } \\
\hline Male & 322 & $16.5(2.7)$ & $16.5(9.0-25.0)$ & 0.32 \\
\hline Female & 314 & $16.7(2.7)$ & $17.0(8.0-26.0)$ & \\
\hline \multicolumn{5}{|l|}{ Refractive error group (right eye) } \\
\hline Hyperopia $\left(\mathrm{SE}^{\star} \geqslant 1.0 \mathrm{D}\right)$ & 84 & $16.7(2.5)$ & $17.0(9.0-21.0)$ & 0.57 \\
\hline Emmetropia $\left(-0.5 \mathrm{D}<\mathrm{SE}^{\star}<+1.0 \mathrm{D}\right)$ & 163 & $16.7(2.9)$ & $17.0(10.0-26.0)$ & \\
\hline Low myopial $\left.-3.0 \mathrm{D}<\mathrm{SE}^{*} \leqslant-0.5 \mathrm{D}\right)$ & 233 & $16.4(2.8)$ & $17.0(8.0-25.0)$ & \\
\hline High myopia $\left(\mathrm{SE}^{*} \leqslant-3 . \mathrm{OD}\right)$ & 156 & $16.7(2.5)$ & $17.0(11.0-24.0)$ & \\
\hline
\end{tabular}


variability of clinical tonometry would affect all subjects equally. ${ }^{6}$ Similar IOP in children, 9-12 years, using pneumatotonometer $(17.3 \mathrm{~mm} \mathrm{Hg})^{4}$ or Goldmann tonometer $(16.6 \mathrm{~mm} \mathrm{Hg})^{5}$ have also been found. Variations in IOP with race and iris colour $^{11}$ are controlled for in this Chinese sample. As expected systolic and diastolic blood pressure ${ }^{10}$ were associated with $\mathrm{IOP}^{19}$ thus providing an indirect measure of the reliability of our IOP readings. Unfortunately, corneal thickness, which may influence $\mathrm{IOP}^{20}$ was not assessed. Also, because of time limitations, only one IOP measurement was taken per eye.

Various definitions of myopia, study population composition, design, and sample sizes limits comparisons with other studies. IOP and myopia ( $\mathrm{SE} \leqslant-0.5 \mathrm{D}$ ) was not associated in Chinese children aged 6-7 years, ${ }^{21}$ although a later casecontrol study comparing myopes with hyperopes ( $\mathrm{SE} \geqslant+1.0$ D) found the opposite. ${ }^{3}$ In a Western country, mean IOP was higher in children with myopia $(17.8 \mathrm{~mm} \mathrm{Hg})$ than emmetropia $(17.1 \mathrm{~mm} \mathrm{Hg}), \mathrm{p}<0.1 .^{4}$ Longitudinal studies evaluating IOP in myopia development and progression have also shown variable results. Children (9-12 years) with high baseline IOP had higher myopia progression rates over 2 years, ${ }^{5}$ yet a later study suggested no effect of baseline IOP on end refraction or progression rates. ${ }^{22}$ In another study, no significant differences in mean IOP were found in children who remained emmetropic ( $14.7 \mathrm{~mm} \mathrm{Hg}$ ) compared with those who became myopic $(13.9 \mathrm{~mm} \mathrm{Hg}){ }^{7}$ Change in IOP was not correlated with change in refraction in preexisting myopes, the investigators concluding that continued myopia development is not the result of high IOP. ${ }^{6}$

Chinese children with higher myopia have longer AL. ${ }^{16}{ }^{23}$ However, the IOP-AL relation in children has been evaluated by few studies. In a study of 49 Danish children, IOP and AL were not related at baseline (AL $24.67 \mathrm{~mm}$ for IOP $>16 \mathrm{~mm} \mathrm{Hg} v 24.53 \mathrm{~mm}$ for IOP $\leqslant 16 \mathrm{~mm} \mathrm{Hg}),{ }^{5}$ although greater mean change in $\mathrm{AL}(0.65 \mathrm{~mm})$ over 2 years was documented in those with higher baseline IOP. Higher IOP and longer AL were found in undergraduate students with myopia (15.49 mm Hg, $24.61 \mathrm{~mm}$ ) compared with hypermetropes $(13.91 \mathrm{mmHg}, 22.53 \mathrm{~mm})$, but not compared with emmetropes (14.74 mm Hg, $23.40 \mathrm{~mm}){ }^{24}$ In 67 anisometropic Chinese children (8-14 years), IOP was not correlated with AL or refractive error differences. ${ }^{23}$

Data evaluating IOP and myopia in Chinese adults are scarce. In Asian-Africans, but not European subjects, associations between IOP and refractive error have been found. ${ }^{13}$ In white populations, the relation between IOP and refractive error ${ }^{10}$ or myopia ${ }^{12} 25$ has been documented, although hyperopia may be associated with the risk of ocular hypertension..$^{25}$ Extrapolating our results to an adult population would be erroneous, and further longitudinal studies assessing whether IOP rises after the onset of myopia would be interesting. Glaucoma remains the important link between myopia and IOP in adults. IOP is an important risk factor of, but is not synonymous with, glaucoma. Myopia is associated with open angle glaucoma in white adults, ${ }^{12}{ }^{25}$ with approximately threefold significantly increased odds in moderate to high myopes. ${ }^{12}$ In these studies, glaucoma was diagnosed independent of IOP, suggesting that predisposition to glaucoma in myopes may not be dependent on mechanisms related to high pressures. In a recent study of Singaporean Chinese, open angle glaucoma was established as the commonest form, ${ }^{26}$ but glaucoma risk factors in Chinese adults are yet to be reported.

In summary, our results suggest that elevated IOP and myopia may not be linked in Chinese children and question the value of ocular hypotensives such as timolol in retarding myopia progression. Longitudinal studies to further assess our findings are warranted.

\section{Authors' affiliations}

A J Lee, S-M Saw, Department of Community, Occupational and Family Medicine, National University of Singapore, 16 Medical Drive,

Singapore 117597, Republic of Singapore

G Gazzard, D T H Tan, Singapore National Eye Centre, (SNEC), 11

Third Hospital Avenue, Singapore 168751, Republic of Singapore

G Gazzard, A Cheng, D T H Tan, Singapore Eye Research Institute,

(SERI) 5th Level, SNEC, 11 Third Hospital Avenue, Singapore 168751, Republic of Singapore

G Gazzard, Institute of Ophthalmology, 11-43 Bath Street, London ECIV 9EL, UK

Correspondence to: Dr Seang-Mei Saw, Department of Community, Occupational and Family Medicine, National University of Singapore, 16 Medical Drive, Singapore 117597, Republic of Singapore;

cofsawsm@nus.edu.sg

Accepted for publication 2 July 2003

Grant support: National Medical Research Council (NMRC), NMRC/ 0695/2002, Singapore.

Commercial relationships: None.

\section{REFERENCES}

1 Saw SM, Katz J, Schein OD, et al. Epidemiology of myopia. Epidemiol Rev 1996;18:175-87

2 Pruett RC. Progressive myopia and intraocular pressure: what is the linkage? A literature review. Acta Ophthalmol Suppl 1988;185:117-27.

3 Edwards MH, Brown B. Intraocular pressure in a selected sample of myopic and non-myopic Chinese children. Optom Vis Sci 1993;70:15-17.

4 Quinn GE, Berlin JA, Young TL, et al. Association of intraocular pressure and myopia in children. Ophthalmology 1995; 102:180-5.

5 Jensen $\mathbf{H}$. Myopia progression in young school children and intraocular pressure. Doc Ophthalmol 1992;82:249-55.

6 Edwards MH, Brown B. IOP in myopic children: the relationship between increases in IOP and the development of myopia. Ophthalmic Physiol Opt 1996; 16:243-6.

7 Goss DA, Caffey TW. Clinical findings before the onset of myopia in youth: 5 . Intraocular pressure. Optom Vis Sci 1999:76:286-91.

8 Jensen $\mathrm{H}$. Timolol maleate in the control of myopia. A preliminary report. Acta Ophthalmol Suppl 1988;185:128-9.

9 Hosaka A. Myopia prevention and therapy. The role of pharmaceutical agents. Japanese studies. Acta Ophthalmol Suppl 1988;185:130-1.

10 Klein BEK, Klein R, Linton KL. Intraocular pressure in an American community, the Beaver Dam Eye Study. Invest Ophthalmol Vis Sci 1992;33:2224-8.

11 Weih LM, Mukesh BN, McCarty CA, et al. Asssociation of demographic, familial, medical, and ocular factors with intraocular pressure. Arch Ophthalmol 2001;119:875-80.

12 Mitchell P, Hourihan F, Sandbach J, et al. The relationship between glaucoma and myopia. Ophthalmology 1999;106:2010-15.

13 David $\mathbf{R}$, Zangwill LM, Tessler $Z$, et al. The correlation between intraocular pressure and refractive status. Arch Ophthalmol 1985;103:1812-15.

14 Daubs JG, Crick RP. Effect of refractive error on the risk of ocular hypertension and open-angle glaucoma. Trans Ophthalmol Soc UK $1981 ; 101: 121-16$

15 Kragha IK. Normal intraocular pressures. Glaucoma 1987;9:89-93.

16 Saw SM, Chua WH, Hong CY, et al. Nearwork in early-onset myopia. Invest Ophthalmol Vis Sci 2002;43:332-9.

17 STATA Statistical Software: Release 7.0. College Station, TX: Stata Corp, 2001.

18 Eisenberg DL, Sherman BG, McKeown, et al. Tonometry in adults and children. A manometric evaluation of pneumatotonometry, applanation and tonopen in vitro and in vivo. Ophthalmology 1998;105:1173-81.

19 McLeod SD, West SK, Quigley HA, et al. A longitudinal study of the relationship between intraocular and blood pressures. Invest Ophthalmol Vis Sci 1990;31:2361-6.

20 Foster PJ, Baasanhu J, Alsbirk PH, et al. Central corneal thickness and intraocular pressure in a Mongolian population. Ophthalmology 1998; 105:969-73

21 Edwards MH, Chun CY, Leung SS. Intraocular pressure in an unselected sample of 6- to 7-year-old Chinese children. Optom Vis Sci 1993;70:198-200.

22 Jensen H. Myopia in teenagers. An eight year follow-up study on myopia progression and risk factors. Acta Ophthalmol Scand 1995;73:389-93.

23 Lee SM, Edwards MH. Intraocular pressure in anisometropic children. Optom Vis Sci 2000;77:675-9.

24 Tomlinson A, Phillips Cl. Applanation tension and axial length of the eyeball. Br J Ophthalmology 1970:54:548-53.

25 Wong TY, Klein BEK, Klein B, et al. Refractive errors, intraocular pressure, and glaucoma in a white population. Ophthalmology 2003;110:211-17.

26 Foster PJ, Oen FT, Machin D, et al. The prevalence of glaucoma in Chinese residents of Singapore: a cross-sectional population survey of the Tanjong Pajar district. Arch Ophthalmol 2000;118:1105-11. 